\title{
La Crosse Virus Disease in the United States, 2003-2019
}

\author{
Grace M. Vahey, ${ }^{1,2}$ Nicole P. Lindsey, ${ }^{1}$ J. Erin Staples, ${ }^{1}$ and Susan L. Hills ${ }^{1 \star}$ \\ ${ }^{1}$ Arboviral Diseases Branch, Centers for Disease Control and Prevention, Fort Collins, Colorado; ${ }^{2}$ Epidemic Intelligence Service, Centers for Disease \\ Control and Prevention, Atlanta, Georgia
}

\begin{abstract}
La Crosse virus (LACV) is an arthropod-borne virus that can cause a nonspecific febrile illness, meningitis, or encephalitis. We reviewed U.S. LACV surveillance data for 2003-2019, including human disease cases and nonhuman infections. Overall, 318 counties in 27 states, principally in the Great Lakes, mid-Atlantic, and southeastern regions, reported LACV activity. A total of 1,281 human LACV disease cases were reported, including 1,183 (92\%) neuroinvasive disease cases. The median age of cases was 8 years (range: 1 month-95 years); 1,130 (88\%) were aged $<18$ years, and 754 $(59 \%)$ were male. The most common clinical syndromes were encephalitis $(N=960 ; 75 \%)$ and meningitis $(N=219$, $17 \%)$. The case fatality rate was $1 \%(N=15)$. A median of 74 cases (range: $35-130)$ was reported per year. The average annual national incidence of neuroinvasive disease cases was 0.02 per 100,000 persons. West Virginia, North Carolina, Tennessee, and Ohio had the highest average annual state incidences $(0.16-0.61$ per 100,000$)$, accounting for $80 \%(N=1,030)$ of cases. No animal LACV infections were reported. Nine states reported LACV-positive mosquito pools, including three states with no reported human disease cases. La Crosse virus is the most common cause of pediatric neuroinvasive arboviral disease in the United States. However, surveillance data likely underestimate LACV disease incidence. Healthcare providers should consider LACV disease in patients, especially children, with febrile illness, meningitis, or encephalitis in areas where the virus circulates and advise their patients on ways to prevent mosquito bites.
\end{abstract}

\section{INTRODUCTION}

La Crosse virus (LACV) is an arthropod-borne virus (arbovirus) in the California serogroup and the family Peribunyaviridae. ${ }^{1}$ La Crosse virus is maintained in its natural transmission cycle between mosquito vectors, primarily the eastern tree hole mosquito Aedes triseriatus, and small mammal amplifying hosts, including chipmunks and gray squirrels. ${ }^{2,3}$ Humans are considered incidental or dead-end hosts.

The majority of LACV infections are asymptomatic. ${ }^{4}$ The incubation period for LACV disease is 5-15 days. The spectrum of clinical disease ranges from nonspecific febrile illness to meningitis and encephalitis. Long-term neurologic sequelae, including recurrent seizures, hemiparesis, and cognitive abnormalities have been reported, but death following LACV disease is uncommon. ${ }^{5-7}$ There is no specific treatment of, and no vaccine to prevent, LACV disease.

La Crosse virus was first isolated in 1964 from brain tissue of a child who had been admitted to a La Crosse, Wisconsin, hospital several years earlier with meningoencephalitis and subsequently died. ${ }^{5}$ La Crosse virus disease has only been reported in the United States, with the majority of cases occurring in states in the Great Lakes, mid-Atlantic, and southeastern regions of the country. ${ }^{6}$ La Crosse virus is recognized as the most common cause of pediatric neuroinvasive arboviral disease in the United States. ${ }^{7}$

Two previous publications have included information on LACV disease cases in summaries of neuroinvasive arboviral disease for 1999-2007 and 2003-2012. ${ }^{7,8}$ However, the latter report focused on pediatric neuroinvasive LACV disease, and neither included non-neuroinvasive disease or nonhuman data. Since 2003, LACV disease cases and nonhuman infection data have been reported to ArboNET, the national arboviral disease surveillance system. We reviewed national LACV surveillance data from ArboNET for 2003-2019 to describe

${ }^{*}$ Address correspondence to Susan L. Hills, Arboviral Diseases Branch, Division of Vector-Borne Diseases, Centers for Disease Control and Prevention, 3156 Rampart Rd, Fort Collins, CO 80521. E-mail: shills@cdc.gov epidemiologic characteristics of reported human disease cases and nonhuman infections.

\section{MATERIALS AND METHODS}

Data source and case definitions. We identified confirmed and probable cases of human LACV disease reported to ArboNET from 2003 to 2019. State health departments report human LACV disease cases to ArboNET based on standardized case definitions, which include clinical and laboratory criteria. ${ }^{9}$ A clinically compatible case of neuroinvasive disease is defined as a person with encephalitis, meningitis, acute flaccid paralysis, or other acute signs of central or peripheral neurologic dysfunction as documented by a physician in the absence of a more likely clinical explanation. A non-neuroinvasive case is defined as a person with fever and without neuroinvasive disease.

According to the current case definition, a confirmed case must have one or more of the following laboratory criteria: 1) demonstration of LACV, antigen, or nucleic acid in tissue, blood, cerebrospinal fluid (CSF), or other body fluid; 2) 4-fold or greater change in LACV quantitative antibody titers in paired sera; 3) LACV immunoglobulin (Ig)M antibodies in serum with confirmatory LACV-neutralizing antibodies in the same or later specimen; and 4) LACV IgM antibodies in CSF and a negative IgM result for other endemic arboviruses. The laboratory criterion for a probable case is LACV IgM antibodies in CSF or serum but with no other testing. ${ }^{9}$

Nonhuman data, including data on veterinary disease cases and infections in mosquitoes, dead birds, and sentinel animals, are reported to ArboNET voluntarily. Nonhuman arboviral surveillance is performed variably across jurisdictions. Data typically reported to ArboNET for nonhuman arboviral infections include species, state and county of collection, and date of symptom onset or specimen collection.

Data analysis. We extracted human disease data, including state and county of residence, age, sex, race, ethnicity, clinical syndrome, date of illness onset, hospitalization, mortality, nonhuman data on species type, and state and county of collection. Categorical variables were described as counts 
and proportions, and continuous variables were described as medians and ranges. We defined pediatric cases as those in children and adolescents aged $<18$ years. Incidence rates were calculated using annual population estimates from the U.S. Census Bureau and neuroinvasive disease case counts, which are considered the most accurate indicator of LACV activity in humans because of the associated morbidity. ${ }^{10}$

We analyzed data using Excel for Office 365 (Microsoft Corp., Redmond, WA) and SAS statistical software version 9.4 (SAS Institute, Cary, NC). Maps were made using ArcGIS version 10.7.1 (Esri, Redlands, CA).

\section{RESULTS}

During 2003-2019, 318 counties in 27 states reported human or nonhuman LACV data to ArboNET. A total of 1,281 human LACV disease cases were reported, including 909 (71\%) confirmed and 372 (29\%) probable cases. Among the 1,281 total cases, 1,183 (92\%) were neuroinvasive disease cases, and 98 (8\%) were non-neuroinvasive disease cases. The most common clinical syndromes were encephalitis $(N=960 ; 75 \%)$ and meningitis $(N=219 ; 17 \%)$ (Table 1$)$.

Human LACV disease cases were reported from 24 states. Four states reported $80 \%$ of all cases: Ohio ( $N=340 ; 27 \%)$, North Carolina ( $N=297 ; 23 \%)$, West Virginia $(N=209$; $16 \%)$, and Tennessee ( $N=184 ; 14 \%)$. In total, 302 counties in these 24 states reported cases (Figure 1). Half of all cases were reported from 26 (9\%) counties, which were in North Carolina, Ohio, Tennessee, and West Virginia, and $21 \%$ of cases were reported from just five (2\%) of counties, namely Buncombe, Transylvania, and Jackson Counties in North Carolina, Knox County in Tennessee, and Raleigh county in West Virginia. Forty-two (3\%) cases were reported as being acquired out of state, including three that were the only cases reported for that state (one from New York and two from Rhode Island).

A median of 74 LACV disease cases (range: 35-130) were reported per year (Figure 2). The highest numbers of cases were reported in 2011 ( $N=130), 2003(N=120)$, and 2004 $(N=117)$. Cases occurred from March through December, but 1,256 (98\%) cases had onset of illness from June through October, with a peak in August (Figure 3).

The median age of LACV disease patients was 8 years (range: 1 month-95 years). Pediatric patients (aged $<18$ years) made up $1,130(88 \%)$ of those with LACV disease. When divided into 5-year age groups, patients aged 6-10 years made up the highest proportion (37\%) of all reported LACV disease cases. Seven hundred fifty-four (59\%) patients were male. Of the 1,160 with known race, 1,092 (94\%) were white, and of the 924 with known ethnicity, 894 (97\%) were non-Hispanic or Latino.

Of the $1,160(91 \%)$ of patients with a known hospitalization status, 1,090 (94\%) were hospitalized. The hospitalization rate for those with neuroinvasive disease was $96 \%(1,030 /$ $1,072)$, compared with $68 \%(60 / 88)$ for those with nonneuroinvasive disease. Overall, there were 15 (1\%) deaths; all were patients with neuroinvasive disease and reported clinical syndromes of encephalitis. The median age of patients with a fatal outcome was 7 years (range: 4-86 years). Case fatality ratios (CFRs) were 1.6\% (12/754) for male patients and $0.6 \%(3 / 526)$ for female patients. Nine $(60 \%)$ of the fatal cases were aged from 0 to 9 years, but the CFR was highest for those aged $\geq 80$ years $(3 / 16 ; 19 \%)$.
TABLE 1

Characteristics of La Crosse virus (LACV) disease cases, United States, 2003-2019

\begin{tabular}{|c|c|}
\hline Characteristics & $\begin{array}{c}\text { LACV disease cases ( } N=1,281 \\
\text { No. (\%) }\end{array}$ \\
\hline \multicolumn{2}{|l|}{ Age group (years) } \\
\hline $0-9$ & $766(60)$ \\
\hline $10-19$ & $370(29)$ \\
\hline $20-29$ & $26(2)$ \\
\hline 30-39 & 19 (1) \\
\hline $40-49$ & $19(1)$ \\
\hline $50-59$ & $21(2)$ \\
\hline $60-69$ & $20(2)$ \\
\hline $70-79$ & $23(2)$ \\
\hline$\geq 80$ & $16(1)$ \\
\hline Unknown & $1(<1)$ \\
\hline \multicolumn{2}{|l|}{ Sex } \\
\hline Male & $754(59)$ \\
\hline Female & $526(41)$ \\
\hline Unknown & $1(<1)$ \\
\hline \multicolumn{2}{|l|}{ Race } \\
\hline White & $1,092(85)$ \\
\hline Black or African American & $31(2)$ \\
\hline American Indian or Alaska Native & $20(2)$ \\
\hline Other & 17 (1) \\
\hline Unknown & $121(9)$ \\
\hline \multicolumn{2}{|l|}{ Ethnicity } \\
\hline Non-Hispanic or Latino & $894(70)$ \\
\hline Hispanic or Latino & $30(2)$ \\
\hline Unknown & $357(28)$ \\
\hline \multicolumn{2}{|l|}{ Clinical syndrome } \\
\hline Encephalitis* & $960(75)$ \\
\hline Meningitis & $219(17)$ \\
\hline Other neuroinvasive & $4(<1)$ \\
\hline Non-neuroinvasive & $98(8)$ \\
\hline \multicolumn{2}{|l|}{ Hospitalized } \\
\hline Yes & $1,090(85)$ \\
\hline No & $63(5)$ \\
\hline Unknown & $128(10)$ \\
\hline \multicolumn{2}{|l|}{ Fatality } \\
\hline Yes & $15(1)$ \\
\hline No & $1,237(97)$ \\
\hline Unknown & $29(2)$ \\
\hline
\end{tabular}

*Includes encephalitis or meningoencephalitis.

For this 17-year period, the nationwide average annual incidence of neuroinvasive LACV disease was 0.02 cases per 100,000 persons. Nationwide annual incidence was highest in 2003, 2004, and 2011 (0.04 per 100,000) and lowest in 2009, 2016, and 2019 (0.01 per 100,000). The state with the highest average annual incidence of neuroinvasive LACV disease was West Virginia ( 0.61 cases per 100,000 persons); this incidence was over three times higher than that in any of the next highest incidence states, which were North Carolina (0.18), Tennessee (0.17), and Ohio (0.16) (Figure 4). These four states were consistently among the five states with the highest annual neuroinvasive disease incidence, with the exception of Tennessee, which had the sixth and seventh highest state incidence in 2016 and 2005, respectively.

During 2003-2019, the nationwide average annual incidence of pediatric neuroinvasive disease was 0.08 cases per 100,000 persons aged $<18$ years. Nationwide annual incidence of pediatric neuroinvasive LACV disease was highest in 2011 ( 0.15 per 100,000 persons aged $<18$ years) and lowest in 2016 (0.03 per 100,000 persons aged $<18$ years). The state with the highest average annual incidence of pediatric 


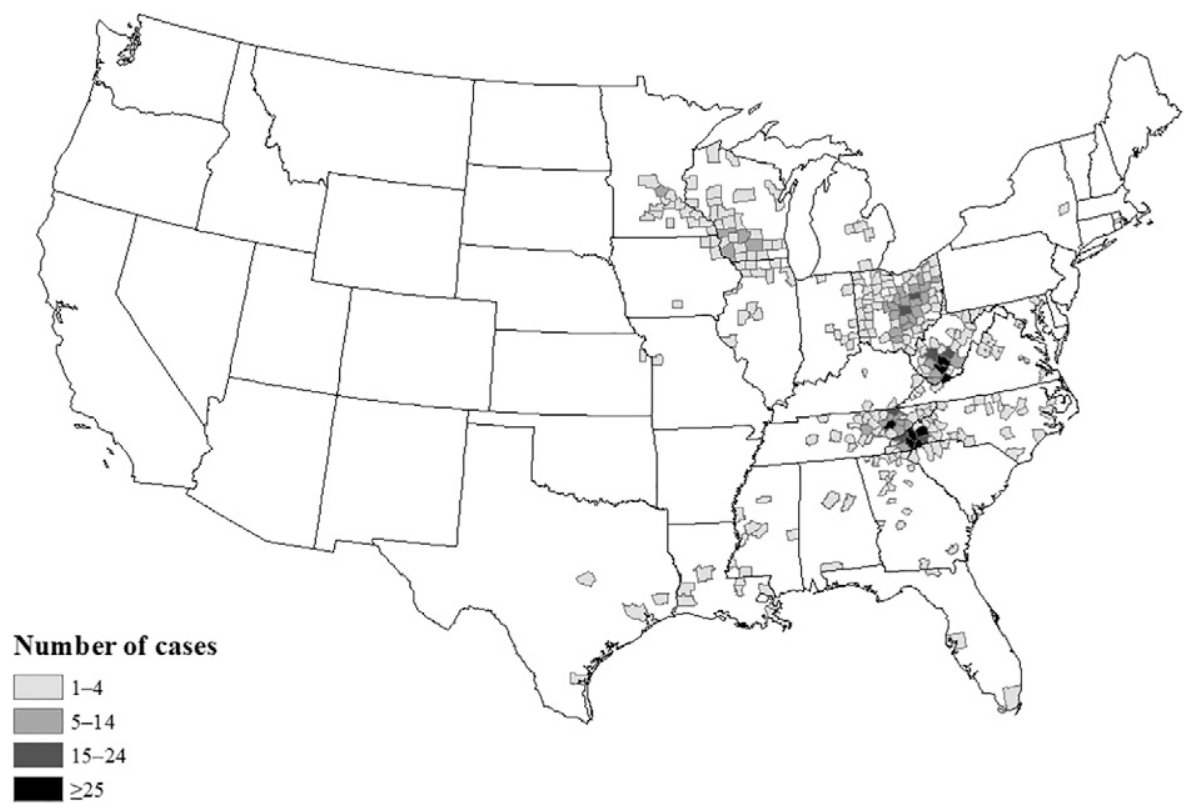

FIGURE 1. Counties reporting human La Crosse virus disease cases, United States, 2003-2019. Cases are reported by county of residence. No cases were reported from Hawaii or Alaska.

neuroinvasive LACV disease was West Virginia ( 2.72 cases per 100,000 persons aged $<18$ years); this incidence was almost four times higher than that in any of the next highest incidence states, which were Tennessee (0.69), Ohio (0.66), and North Carolina (0.59).

No animal LACV infections were reported during 2003-2019. A total of 55 LACV mosquito pool infections were reported from 22 counties in nine states, namely Connecticut, Michigan, Minnesota, North Dakota, New Jersey, New York, Ohio, Texas, and West Virginia (Supplemental Table 1). Six (27\%) of the 22 counties reported both human disease cases and mosquito infections, and 16 (73\%) reported mosquito infections only. Of the 55 positive pools, $31(56 \%)$ were from West Virginia. Mosquito

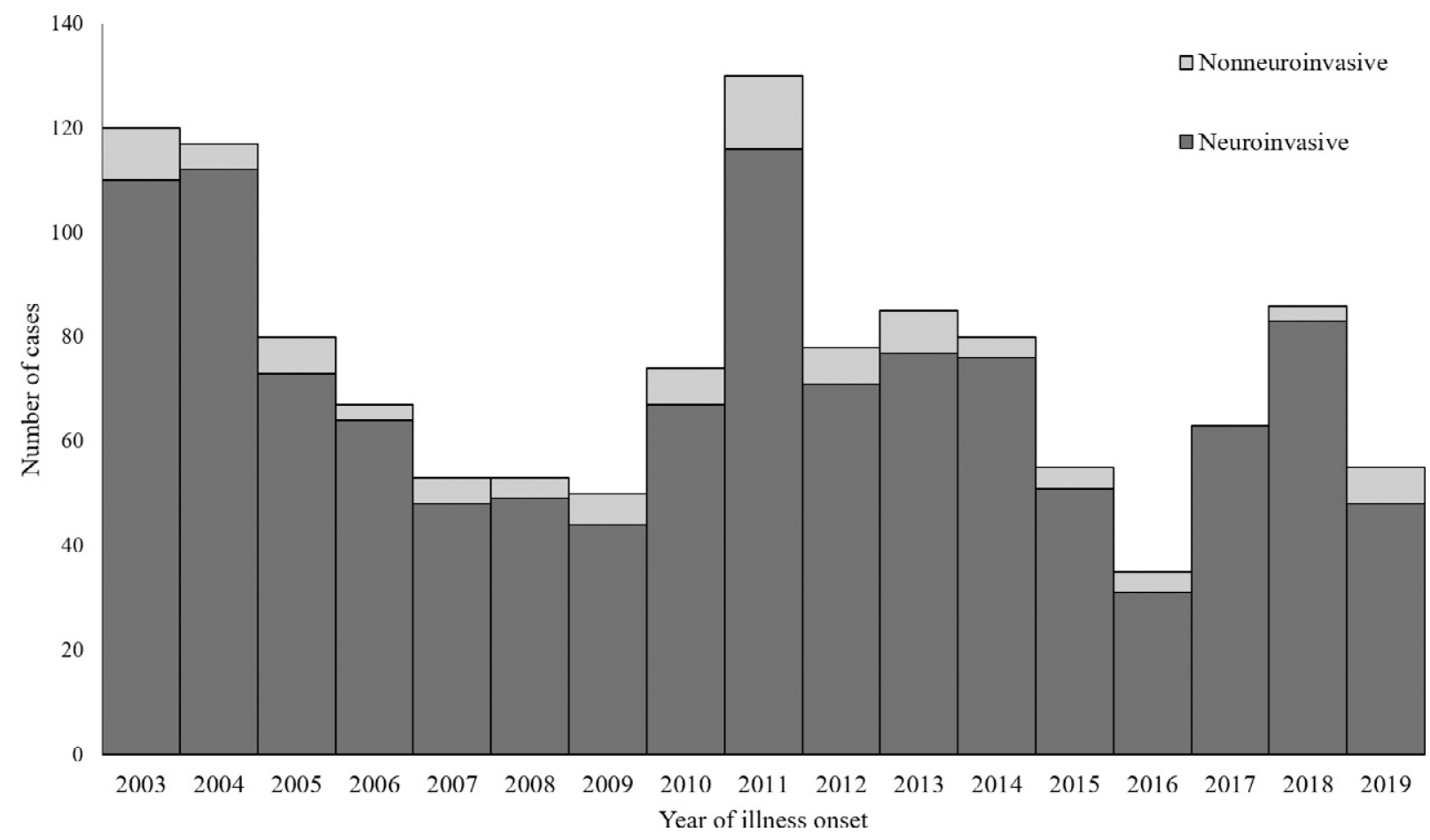

FIGURE 2. La Crosse virus disease cases by year of onset and clinical presentation, United States, 2003-2019. 


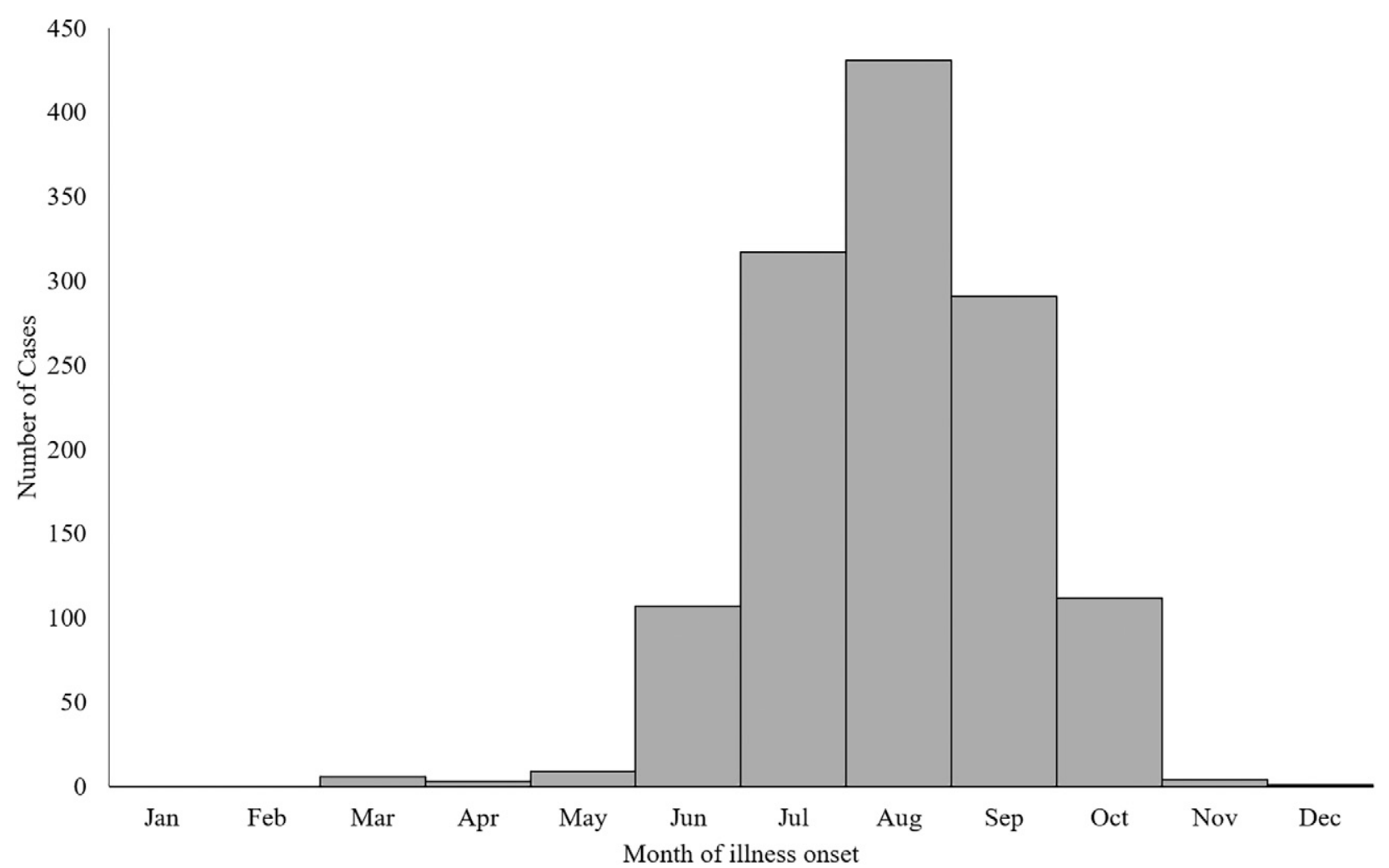

FIGURE 3. La Crosse virus disease cases by month of illness onset, United States, 2003-2019.

infections were reported in three states (Connecticut, North Dakota, and New Jersey) that did not report human disease cases, and from New York, which only reported one human case that was acquired out of state. Infected mosquitoes were collected from May through October, with 47 (85\%) collected from July through September. La Crosse virus-positive pools were from at least seven mosquito species, including 40 (73\%) from any Aedes species, including 15 (27\%) specifically reported as Aedes triseriatus.

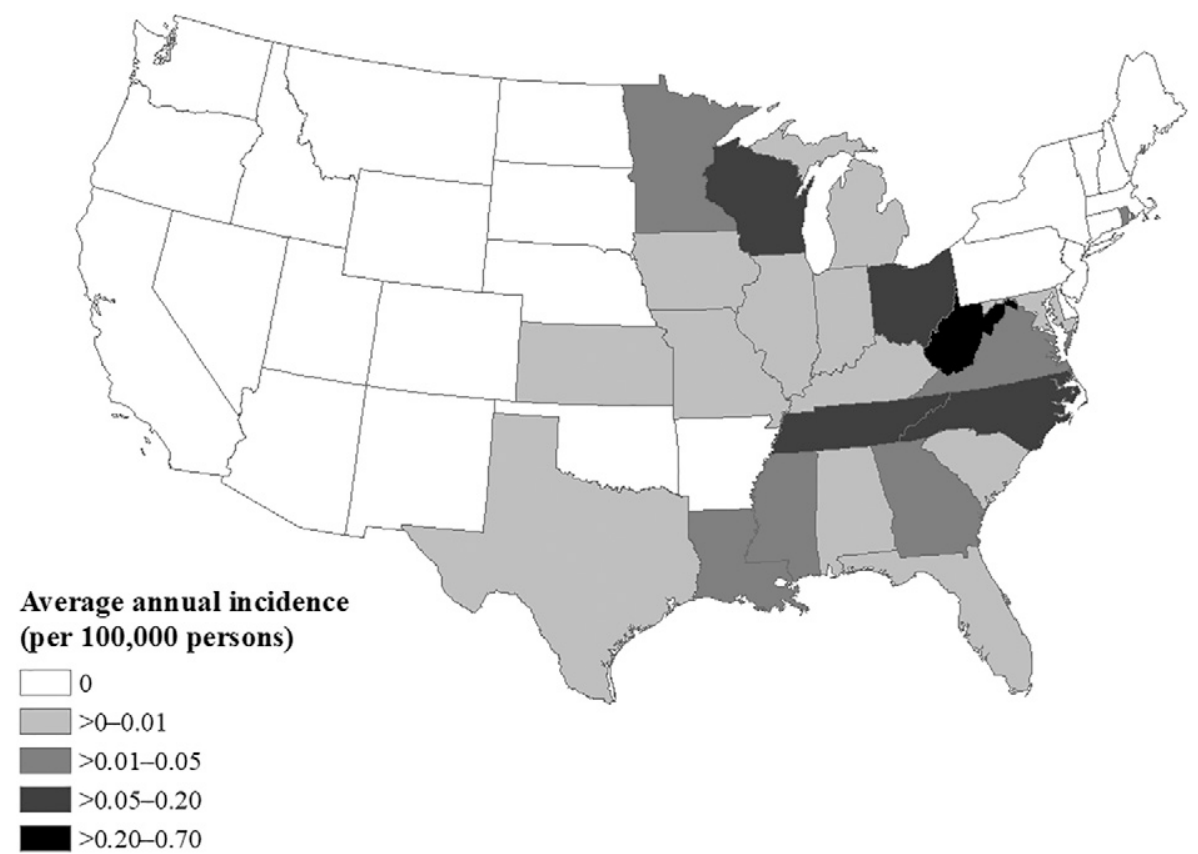

FlGURE 4. Average annual incidence of neuroinvasive La Crosse virus disease by state, United States, 2003-2019. Calculations are based on cases' states of residence. No cases were reported from Hawaii or Alaska. 


\section{DISCUSSION}

During the 17-year period from 2003 to 2019, LACV was an important cause of pediatric neuroinvasive disease in the United States. Among the 1,281 cases of human LACV disease reported to ArboNET, over $92 \%$ were neuroinvasive disease, and almost $90 \%$ were among persons aged $<18$ years. Most (80\%) of LACV disease cases were residents of just four states, including Ohio, North Carolina, West Virginia, and Tennessee. Although most cases required hospitalization, the overall case fatality rate was low.

The predominance of LACV disease cases in children and adolescents is consistent with historical patterns of LACV disease; from 1972 to $1981,>90 \%$ of reported California serogroup encephalitis cases were in persons $<15$ years of age. ${ }^{3,11}$ The reasons for this predisposition are incompletely characterized, but in vitro and animal model studies have demonstrated that there are age-related differences in both innate and adaptive immunity that might allow viral replication and invasion of the central nervous system in younger individuals. $^{12,13}$ The high proportion of cases among children and adolescents is unlike patterns observed with other arboviral diseases in the United States. For example, only about $4 \%$ of reported West Nile virus disease cases occur among children. ${ }^{7}$ Because other common causes of pediatric viral encephalitis, such as enterovirus and herpes simplex virus, are not nationally notifiable conditions, their national incidence cannot be directly compared with that of LACV disease. However, in a review of 6,665 U.S. pediatric encephalitis and meningitis cases occurring during 2011-2014, arboviral etiologies accounted for $0.5 \%$ of cases, compared with $58.4 \%$ and $1.5 \%$ for enterovirus or herpes simplex viruses, respectively. ${ }^{14}$

The geographic distribution of reported LACV disease cases likely reflects the distribution of suitable habitat for vectors and amplifying host species. ${ }^{3}$ The distribution of reported cases also might be influenced by differences in surveillance activity, including clinician awareness and testing practices, by jurisdiction. Additionally, it is thought that LACV transmission risk is highly focal, ${ }^{3,15,16}$ which is reflected in our finding that $9 \%$ of the 302 counties that reported human LACV disease cases reported $50 \%$ of the disease cases. The reason that Connecticut, North Dakota, New Jersey, and New York reported LACV mosquito infections but not locally acquired human disease cases is unknown. It might be because of limited human-mosquito interaction in the locations where the infected mosquitoes were collected, insufficient human testing practices, or differences in testing and reporting practices.

The CFR for LACV disease cases reported to ArboNET is lower than for other neuroinvasive arboviral diseases. ${ }^{3}$ This might reflect that a high proportion of reported LACV disease cases are children, who have lower CFRs for most arboviral diseases. For West Nile virus neuroinvasive disease, which has a higher proportion of reported cases among adults, the overall CFR is about $9 \%$. However, the CFR of the pediatric West Nile virus neuroinvasive disease is $1 \%$, which is similar to the CFR for LACV disease., ${ }^{77}$ Additionally, evidence suggests that only a narrow range of LACV lineage 1 genotypes are associated with fatal outcomes. However, a comprehensive understanding of the reasons for the low LACV CFR is lacking. ${ }^{18,19}$

The findings in this review are subject to several limitations. First, ArboNET is a passive surveillance system, and reporting of LACV disease cases relies on clinician awareness and recognition of potential cases, access to and performance of the appropriate diagnostic test, and positive results being notified to public health authorities. Therefore, diagnosis and reporting are likely incomplete, and the incidence of LACV disease is likely underestimated, particularly for cases of nonneuroinvasive disease because patients are less likely to present for medical evaluation and might not have the appropriate testing performed given the nonspecific symptoms. The high rate of hospitalization of almost $70 \%$ for patients with nonneuroinvasive disease similarly suggests that more severe cases might be more frequently diagnosed and reported. Second, ArboNET does not require information regarding diagnostics performed for other arboviral diseases, and it cannot be determined if all cases have had appropriate confirmatory testing. Subsequently, there is the potential for misclassification due to cross-reactivity with Jamestown Canyon virus, another California serogroup arbovirus, particularly for probable cases where only lgM was detected and differential neutralizing antibody testing was not performed. ${ }^{20}$ Third, case definitions used for surveillance have changed slightly during this time period, which might affect comparability of cases reported over time. ${ }^{9}$ Finally, nonhuman surveillance is highly variable between jurisdictions, and mosquito and animal data are reported voluntarily, limiting the ability to make conclusions about these data or to compare between jurisdictions.

Because arboviral disease transmission depends on multiple complex factors, including weather, zoonotic host and vector abundance, and human behavior, it is very difficult to explain or predict the number of LACV disease cases reported each year. This highlights the importance of continued surveillance to detect outbreaks and identify changing trends to inform public health prevention efforts.

Persons, especially children, living in areas where LACV disease cases or mosquito infections have been reported should take action to prevent mosquito bites, as this is the primary means of preventing LACV infection. This includes using insect repellent, wearing long-sleeved shirts and long pants when outside, having intact screens on windows and doors, using air conditioning, and eliminating mosquito breeding sites by removing outdoor sources of standing water and filling tree holes. Insect repellents registered by the U.S. Environmental Protection Agency should also be used as labeled, noting that some repellents, such as oil of lemon eucalyptus and paramenthane-diol, should not be used on children aged $<3$ years. ${ }^{21}$ Healthcare providers in areas where LACV is endemic should consider LACV disease in patients with febrile illness, meningitis, or encephalitis and a history of mosquito bites.

Received March 10, 2021. Accepted for publication April 25, 2021.

Published online July 19, 2021.

Note: Supplemental table appears at www.ajtmh.org.

Acknowledgments: We thank Sarabeth Mathis for assistance in obtaining data for this report and ArboNET database management as well as staff at local and state health departments for performing surveillance and reporting to ArboNET.

Financial support: This work was completed as part of official duties of staff at the Centers for Disease Control and Prevention.

Disclaimer: The findings and conclusions of this report are those of the authors and do not necessarily represent the official position of the Centers for Disease Control and Prevention.

Authors' addresses: Grace M. Vahey, Nicole P. Lindsey, J. Erin Staples, and Susan L. Hills, Arboviral Diseases Branch, Centers for 
Disease Control and Prevention, Fort Collins, CO, E-mails: gvahey@ cdc.gov, nplindsey@cdc.gov, estaples@cdc.gov, and shills@cdc.gov.

\section{REFERENCES}

1. Hughes HR, Lanciotti RS, Blair CD, Lambert AJ, 2017. Full genomic characterization of California serogroup viruses, genus Orthobunyavirus, family Peribunyaviridae including phylogenetic relationships. Virology 512: 201-210.

2. Tsai TF, 1991. Arboviral infections in the United States. Infect Dis Clin North Am 5: 73-102.

3. Calisher $\mathrm{CH}, 1994$. Medically important arboviruses of the United States and Canada. Clin Microbiol Rev 7: 89-116.

4. Grimstad PR, Barrett CL, Humphrey RL, Sinsko MJ, 1984. Serologic evidence for widespread infection with La Crosse and St. Louis encephalitis viruses in the Indiana human population. Am J Epidemiol 119: 913-930.

5. Thompson WH, Kalfayan B, Anslow RO, 1965. Isolation of California encephalitis group virus from a fatal human illness. $A m ~ J$ Epidemiol 81: 245-253.

6. Harding S, Greig J, Mascarenhas M, Young I, Waddell LA, 2018. La Crosse virus: a scoping review of the global evidence. Epidemiol Infect 14: 1-13.

7. Gaensbauer JT, Lindsey NP, Messacar K, Staples JE, Fischer M, 2014. Neuroinvasive arboviral disease in the United States: 2003 to 2012. Pediatrics 134: e642-e650.

8. Reimann CA, Hayes EB, DiGuiseppi C, Hoffman R, Lehman JA, Lindsey NP, Campbell GL, Fischer M, 2008. Epidemiology of neuroinvasive arboviral disease in the United States, 1999-2007. Am J Trop Med Hyg 79: 974-979.

9. Centers for Disease Control and Prevention, 2015. Arboviral Diseases, Neuroinvasive and Non-neuroinvasive 2015 Case Definition. Available at: https://wwwn.cdc.gov/nndss/conditions/ arboviral-diseases-neuroinvasive-and-non-neuroinvasive/ case-definition/2015/. Accessed November 18, 2019.

10. United States Census Bureau, 2018. State Population Totals. Available at: census.gov. Accessed November 18, 2019.

11. Kappus KD, Monath TP, Kaminski RM, Calisher CH, 1983. Reported encephalitis associated with California serogroup virus infections in the United States, 1963-1981. Prog Clin Biol Res 123: 31-41.
12. Taylor KG, Woods TA, Winkler CW, Carmody AB, Peterson KE, 2014. Age-dependent myeloid dendritic cell responses mediate resistance to La Crosse virus-induced neurological disease. $J$ Virol 88: 11070-11079.

13. Winkler CW, Myers LM, Woods TA, Carmody AB, Taylor KG, Peterson KE, 2017. Lymphocytes have a role in protection, but not in pathogenesis, during La Crosse virus infection in mice. J Neuroinflammation 14: 62.

14. Hasbun R, Wootton SH, Rosenthal N, Balada-Llasat JM, Chung J, Duff S, Bozzette S, Zimmer L, Ginocchio CC, 2019. Epidemiology of meningitis and encephalitis in infants and children in the United States, 2011-2014. Pediatr Infect Dis J 38: 37-41.

15. Erwin PC, Jones TF, Gerhardt RR, Halford SK, Smith AB, Patterson LE, Gottfried KL, Burkhalter KL, Nasci RS, Schaffner W, 2002. La Crosse encephalitis in eastern Tennessee: clinical, environmental, and entomological characteristics from a blinded cohort study. Am J Epidemiol 155: 1060-1065.

16. Byrd BD, Williams CJ, Staples JE, Burkhalter KL, Savage HM, Doyle MS, 2018. Notes from the field: spatially associated coincident and noncoincident cases of La Crosse encephalitis North Carolina, 2002-2017. MMWR Morb Mortal Wkly Rep 67: 1104-1105.

17. Hayes EB, Komar N, Nasci RS, Montgomery SP, O'Leary DR, Campbell GL, 2005. Epidemiology and transmission dynamics of West Nile virus disease. Emerg Infect Dis 11: 1167-1173.

18. Lambert AJ, Fryxell RT, Freyman K, Ulloa A, Velez JO, Paulsen D, Lanciotti RS, Moncayo A, 2015. Comparative sequence analyses of La Crosse virus strain isolated from patient with fatal encephalitis, Tennessee, USA. Emerg Infect Dis 21: 833-836.

19. Huang C, Thompson WH, Karabatsos N, Grady L, Campbell WP, 1997. Evidence that fatal human infections with La Crosse virus may be associated with a narrow range of genotypes. Virus Res 48: $143-148$.

20. Pastula DM, Hoang Johnson DK, White JL, Dupuis AP 2nd, Fischer M, Staples JE, 2015. Jamestown Canyon Virus Disease in the United States -2000-2013. Am J Trop Med Hyg 93: 384389.

21. Committee on Infectious Diseases, American Academy of Pediatrics, Kimberlin DW, Brady MT, Jackson MA, Long SS, 2018. Repellents for Use on Skin. Red Book: Report of the Committee on Infectious Diseases. Itasca, IL: American Academy of Pediatrics, 197-199. 